Shedding Phenomenon of Ventilated Partial Cavitation around an Underwater Projectile

This article has been downloaded from IOPscience. Please scroll down to see the full text article.

2012 Chinese Phys. Lett. 29014601

(http://iopscience.iop.org/0256-307X/29/1/014601)

View the table of contents for this issue, or go to the journal homepage for more

Download details:

IP Address: 159.226.231.70

The article was downloaded on 18/04/2013 at 10:45

Please note that terms and conditions apply. 


\title{
Shedding Phenomenon of Ventilated Partial Cavitation around an Underwater Projectile
}

\author{
WANG Yi-Wei(王一伟) ${ }^{1}$, HUANG Chen-Guang(黄晨光 $)^{1}$, DU Te-Zhuan(杜特专 $)^{1}$, WU Xian-Qian(吴先前 $)^{1}$, \\ FANG Xin(方新 $)^{2}$, LIANG Nai-Gang(梁乃刚 $)^{2}$, WEI Yan-Peng(魏延鹏 $)^{1 * *}$ \\ ${ }^{1}$ Key Laboratory of Hydrodynamics and Ocean Engineering, Institute of Mechanics, \\ Chinese Academy of Sciences, Beijing 100190 \\ ${ }^{2}$ The State Key Laboratory of Nonlinear Mechanics, Institute of Mechanics, \\ Chinese Academy of Sciences, Beijing 100190
}

(Received 2 August 2011)

\begin{abstract}
A new shedding phenomenon of ventilated partial cavitations is observed around an axisymmetric projectile in a horizontal launching experiment. The experiment system is established based on SHPB launching and high speed photography. A numerical simulation is carried out based on the homogeneous mixture approach, and its predicted evolutions of cavities are compared with the experimental results. The cavity breaks off by the interaction between the gas injection and the re-entry jet at the middle location of the projectile, which is obviously different from natural cavitation. The mechanism of cavity breaking and shedding is investigated, and the influences of important factors are also discussed.
\end{abstract}

PACS: 46.40.Cd, 47.55.Dp DOI:10.1088/0256-307X/29/1/014601

Artificial gas injection is an effective method to reduce friction drag and optimize flow for high-speed underwater vehicles. ${ }^{[1,2]}$ There are usually three ways to achieve this. First, huge amounts of noncondensable gas are injected into the cavity to form a supercavity. ${ }^{[3,4]}$ Second, bubbles of noncondensable gas are injected into the liquid turbulent boundary layer to form a gas layer, which can reduce the friction drag. ${ }^{[5]}$ Third, gas is injected to the partial cavity, which can also increase stability.

For the third method mentioned above, re-entry jets are often observed for natural partial cavities, flowing upstream along the flow axis and leading to quasi-periodical evolutions such as breaking, shedding and internal collapse. Many analyses have been presented by using cavitation tunnels and other relevant measuring equipment. Among them, Kubota et al. ${ }^{[6]}$ described the unsteady flow field of the cloud cavitation, and their results indicated that the shedding cavitation cloud was comprised of large scale eddies. Le et $a l .{ }^{[7]}$ measured the collapse pressure of the shedding cavity. Callenaere ${ }^{[8]}$ investigated the re-entrant jet instability and the associated cloud cavitation on a plano-convex hydrofoil, and carried out a variety of different classifications and analyses on these phenomena. Correspondingly, in Ceccio's review paper, ${ }^{[2]}$ it was mentioned that gas injection can prevent strong re-entry jets and cavity shedding ideally. There is also research on stable ventilated partial cavitations. For example, Kunz et al. ${ }^{[9]}$ developed a multi-phase CFD method to analyze natural and ventilated cavitations, and their results indicate that the ventilated partial cavitation exhibits similar dynamic behavior to natural cavities but tends to be more stable. A similar approach for the three-component model has been reported by Ji et al. ${ }^{[10]}$ Kopriva et al. ${ }^{[11]}$ carried out an experimental study of hydrofoil drag reduction by ventilated partial cavitation in both steady and unsteady flows, in which effective drag reduction and sharp increasing of lift to drag ratio were achieved. Lay et $a l .{ }^{[12]}$ conducted a large-scale experiment of the ventilated cavity formed downstream of a backward-facing step, and reported that the produced stable cavity can reduce the skin drag by more than $95 \%$ over the extent of the cavity, including the cavity closure.

Previous research has mainly focused on stable cavitation, especially supercavitation, however, unstable cloud cavities can be created from re-entry jets in ventilated cavitations. In this Letter, unsteady characteristics of the ventilated partial cavitation around an axisymmetric projectile are investigated. A cloud cavity shedding phenomenon is observed in the experiment, and its mechanism is analyzed.

The experimental system is a split Hopkinson pressure bar (SHPB) based launching device, ${ }^{[13]}$ which can transiently accelerate the projectile and cause little disturbance in water tank. The details of the system has been reported elsewhere. ${ }^{[14]}$ To create the ventilated cavity, the projectile is designed as a hollow cylinder with a conical head (as shown in Fig. 1). The length of the projectile is $246 \mathrm{~mm}$ and the diameter is $37 \mathrm{~mm}$. There are 16 ventilating ducts in total, distributed at the same distance around the projectile's shoulder. The external diameter of the duct is $1.5 \mathrm{~mm}$

** Corresponding author. Email: weiyanpeng@imech.ac.cn
(c) 2012 Chinese Physical Society and IOP Publishing Ltd 
and the internal diameter is $1 \mathrm{~mm}$.

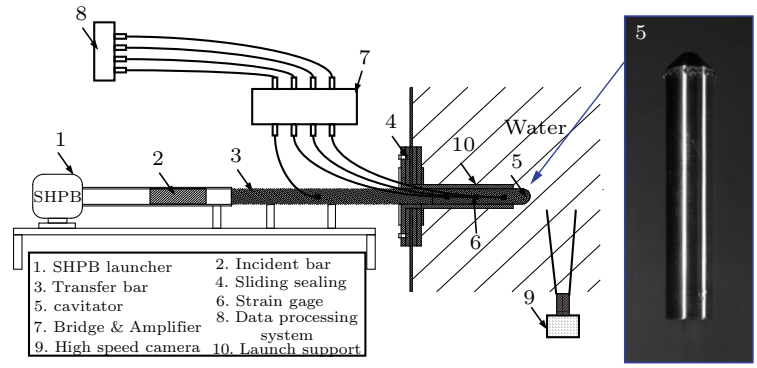

Fig. 1. Launch system and the experimental projectile model.

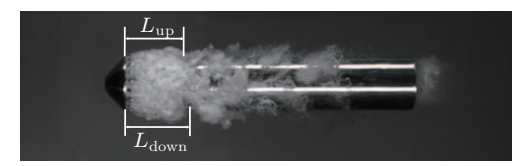

Fig. 2. Typical experimental photograph.

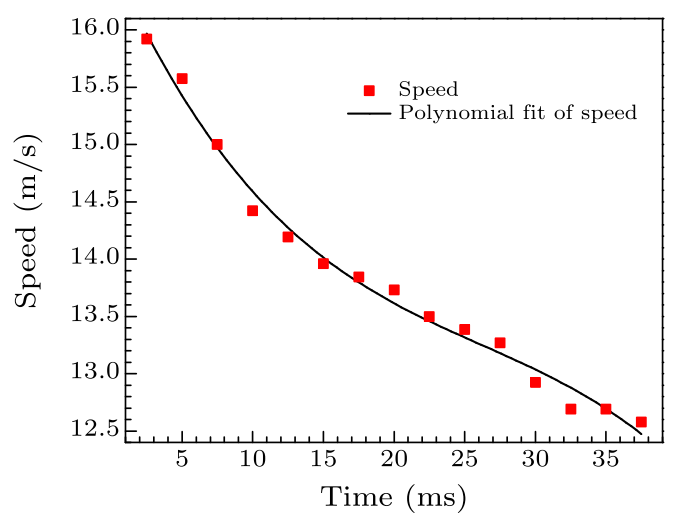

Fig. 3. Time history of the model's speed.

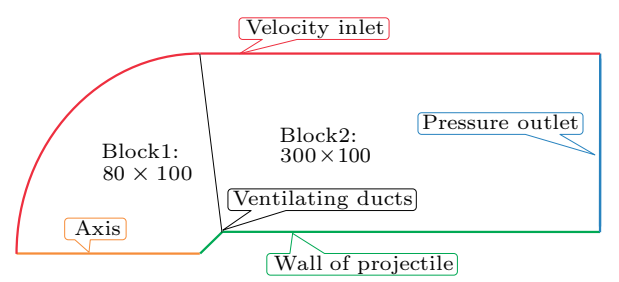

Fig. 4. Computational domain and mesh.

Before launching, the interior of the projectile was full of air with pressure at $1 \mathrm{~atm}$. When the projectile is launched, the pressure drops significantly around the shoulder and the cavitation occurs, then the air inside the projectile injects into the cavity through the duct at a very high speed. A high speed camera capable of capturing $10^{5} \mathrm{fps}$ was used to record the process. A typical photograph gained is shown in Fig. 2. In the image we can clearly see the cavitation with shedding bubbles appearing at the shoulder and tail of the projectile. The length of the cavitation was defined as shown in Fig. 2. In the following, the evolution of the cavitation will be mainly studied based on experimental images and numerical simulation.

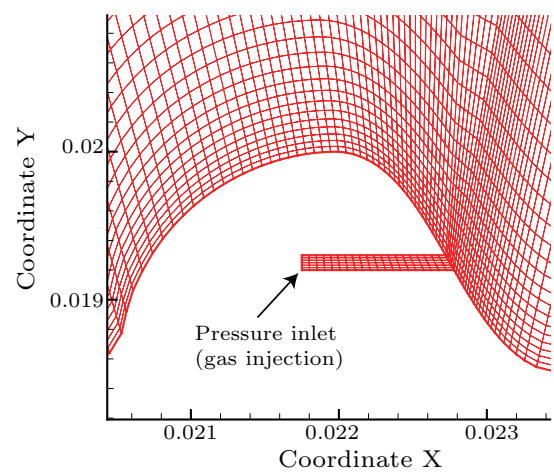

Fig. 5. Mesh and boundary conditions near the ventilating ducts.

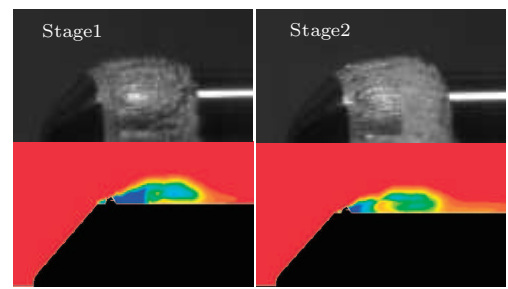

Fig. 6. Typical cavitation patterns in stage 1 and 2 (experimental photographs are shown in the top views, while the numerically predicted results are shown in the bottom views).

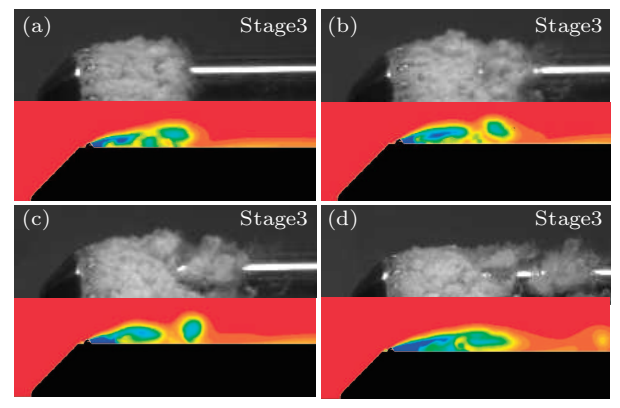

Fig. 7. A typical cavity shedding process of stage 3 .

In a typical experiment, the projectile was launched with an initial speed of $16 \mathrm{~m} / \mathrm{s}$. Through analysis of the obtained images, speed variation can be calculated by a center differential method as shown in Fig. 3. The total pressure of the air inside the projectile is initially $1 \mathrm{~atm}$. The duct is the throat of air injection flow where the Mach number is equal to 1. With a simple aerodynamic calculation, the total pressure at the exit of the duct is about $0.93 \mathrm{~atm}$, and the mass flow rate per duct is approximately $0.15 \mathrm{~g} / \mathrm{s}$.

In order to simulate the motions of water, vapor and noncondensable air, and the interphase change of the first two phases, the homogeneous mixture approach is adopted, in which a classical Singhal model is used to simulate the phase change, and a modified RNG $k-\varepsilon$ model with Reboud correction is used to simulate the turbulence. Details about the numerical 
method can be found in Ref. [15].

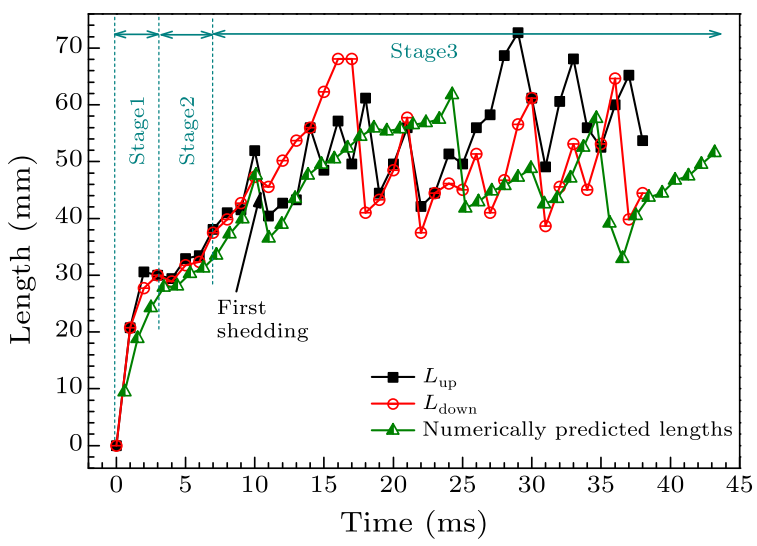

Fig. 8. Comparisons of cavitations lengths between experimental and numerical results.

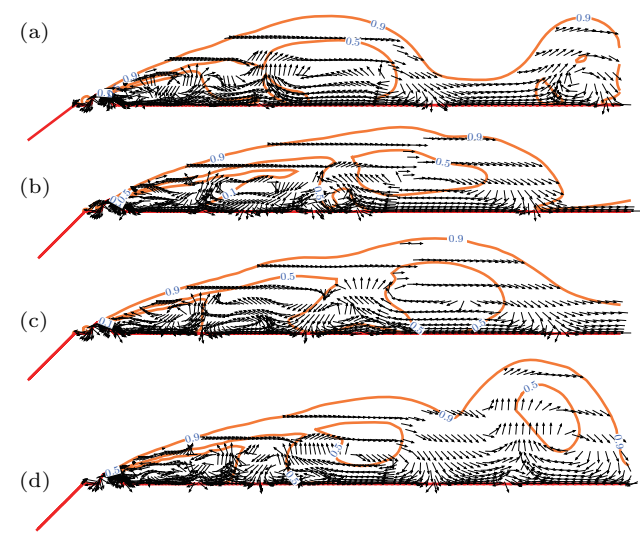

Fig. 9. Velocity fields in cavities in the shedding process.

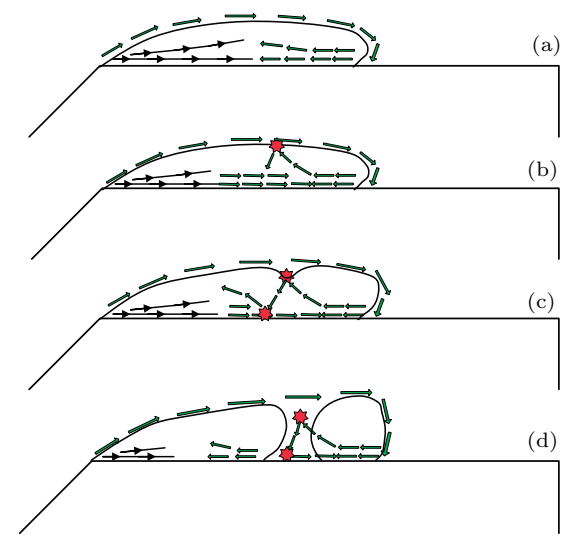

Fig. 10. Dynamic analyses about the shedding phenomenon.

In this study, the computational domain is set to be axisymmetically discretized with block structure grids, which is shown in Fig. 4. The grid near the ventilating duct is shown in Fig. 5, and the pressure inlet condition is applied for the air injection with the total pressure as $0.93 \mathrm{~atm}$. During the period of the experimental observation, the air loss is negligible, so pressure change of air injection is not considered.
From both the experimental and numerical results, it can be seen that cavity shape evolves in three stages: cavity growth and the development of the re-entry jet and its back flow to the projectile shoulder, and the interaction between the gas injection and the re-entry jet, as shown in Figs. 6 and 7. The evolution of the cavity length is shown in Fig. 8 based on the measurement method in Fig. 3. In the first two stages, the cavity lengths above and below the projectile are nearly the same, which indicates that the cavity shape retains its axisymmetric property. During this period the evolution is similar to that of natural cavitation.

A quasi-periodic shedding phenomenon appears in the third stage. A typical evolution of the cavity shedding process is shown in Fig. 7 (both experiment and simulation). The cavity shape in this stage is no longer axisymmetric. The cavity length varies with the time as a result of growth and shedding of the cavity, and lengths above and below the projectile are obviously different. The numerically predicted length also shows a similar value and development trend as the experimental result.

The shedding phenomena observed here are very particular under ventilation conditions. Its mechanism and the difference with natural cavitations are worth noticing. For the shedding process shown in Fig. 7, the numerically predicted evolution of velocity fields is shown in Fig. 9 (contour lines represent the volume fraction of water), and the flow patterns can also be analyzed as schematic diagrams shown in Fig. 10.

As can be seen from Figs. 9(a) and 10(a), before disturbed, the gas injection and re-entry jet both flow close to the projectile wall. When they meet each other, the re-entry jet is pushed away from the projectile.

When the re-entry jet encounters the cavity surface, the pressure arises in the interaction area, which makes the re-entry jet turn around and flow towards the projectile (shown in Figs. 9(b) and 10(b)).

When the re-entry jet meets the gas injection again, a high pressure area is also formed near the projectile wall, which makes the jet turn the flow direction again in a snake-like movement (shown in Figs. 9(c) and $10(\mathrm{c}))$.

As the re-entry jet continues to flow upstream, the two high-pressure zones expand and gradually connect, and the cavity breaks within the region between them. The downstream cavity is within a vortex and will shed with the main flow. At this time, the flow pattern around the upstream cavity is exactly the same as the one shown in Figs. 9(a) and 10(a). The upstream cavity will continue to evolve similarly as described above (shown in Figs. 9(d) and 10(d)).

As described above, it is the interaction between 
the gas injection and the re-entry jet that induces the cavitation to break and shed. Different from the natural cavitation, the cavity usually sheds as a whole caused by the re-entry jets flowing back to the shoulder. Furthermore, we can infer that the breaking location is influenced by the energy ratio of the gas injection to the re-entry jet. Under the experimental condition in this study, the ratio is approximately 1 , which makes the cavity break in the middle position.

In summary, with a projectile launching device based on an SHPB system, a new shedding phenomenon of partial cavitation around the underwater projectile is observed, and the mechanism is investigated based on a numerical simulation with the homogeneous mixture approach. For the shedding phenomenon of the ventilated cavitation presented here, the cavity breaks in the middle location of the projectile quasi-periodically, which is significantly different from natural cavitation. Numerical simulation indicates that the re-entry jet breaks off the cavity from outside towards the projectile after a series of interactions with the gas injection. The breaking location of cavities depends on the energy ratio of the gas injection to the re-entry jet. Numerical methods adopted here can effectively represent the physical phenomena, so they can be further applied to predict unsteady cav- itations with similar conditions.

\section{References}

[1] Brennen C E 1995 Cavitation and Bubble Dynamics (New York: Oxford University)

[2] Ceccio S L 2010 Annu. Rev. Fluid. Mech. 42138

[3] Kuklinski R, Henoch C and Castano J 2001 Fourth International Symposium on Cavitation (California: California Institute of Technology)

[4] Yu K P, Zhou J, J Ming J X and Zhang G 2010 J. Fluid. Eng. T. Asme. 13211303

[5] Merkle C and Deutsch S 1992 Appl. Mech. Rev. 45103

[6] Kubota A, Kato H, Yamaguchi H and Maeda M $1989 \mathrm{~J}$. Fluid. Eng. T. Asme. 111204

[7] Le Q, Franc J and Michel J 1993 J. Fluid. Eng. T. Asme. 115249

[8] Callenaere M, Franc J P, Michel J M and Riondet M 2001 J. Fluid. Mech. 444223

[9] Kunz R F, Boger D A and Stinebring D R 1998 Comput. Fluids 29849

[10] Ji B, Luo X W, Zhang Y, Ran H J, Xu H Y and Wu Y L 2010 Chin. Phys. Lett. 27096401

[11] Kopriva J E, Amromin E L, Arndt R E A, Wosnik M and Kovinskaya S 2007 J. Ship. Res. 51313

[12] Lay K A, Yakushiji R, Makiharju S, Perlin M and Ceccio S L 2010 J. Ship. Res. 54109

[13] Liu K X and Li X D 2006 Chin. Phys. Lett. 233045

[14] Wei Y P, Wang Y W, Fang X, Huang C G and Duan Z P 2011 Chin. Phys. Lett. 28024601

[15] Wang Y W, Huang C G, Du T Z and Liu W W 2011 Chin. J. Hydrodyn. 2648 